\title{
Optimum Assembly Planning for Modular Construction Using BIM and 3D Point Clouds
}

\author{
Mohammad NAHANGI ${ }^{1, *}$, Christopher RAUSCH${ }^{2}$, and Carl HAAS ${ }^{3}$ \\ ${ }^{1}$ Post-Doctoral Fellow and Research Assistant, Department of Civil and Environmental \\ Engineering, University of Waterloo, Canada. \\ ${ }^{2}$ Graduate Research Assistant and Masters Candidate, Department of Civil and Environmental \\ Engineering, University of Waterloo, Canada \\ ${ }^{3}$ Professor, Department of Civil and Environmental Engineering, University of Waterloo, \\ Canada. \\ "Corresponding author's e-mail: mnahangi@uwaterloo.ca
}

\begin{abstract}
Geometric and dimensional deviations often create challenges for component aggregation in the assembly of interchangeable components in modular construction. Although the components are designed interchangeably, once they are fabricated, there are inevitable discrepancies between the designed and built states. Such discrepancies create problems for fitting interchangeable modular components. This paper presents a framework for optimally planning the assembly of interchangeable components based on their as-built state. A 3D point cloud model is captured and the critical interfaces between modules are compared to the original state, integrated in the building information models (BIM), as 3D drawings. The optimization framework is implemented based on two different approaches: (1) minimization of the total deviation for minimizing rework, and (2) intervention of rework by finding the best matching component for each investigated slot. Results show that the method can be effectively used for reducing rework in modular construction by optimum assembly planning.
\end{abstract}

\section{KEYWORDS}

Optimum assembly plan, modular construction, variability, geometric deviation, 3D point clouds, building information model (BIM)

\section{INTRODUCTION}

Although technological and process-related advancements are growing significantly in offsite construction, variabilities in geometry are inevitable and cause challenges for assembly planning. Despite automated production processes used are very accurate, component geometry can deviate from its originally designed state, as secondary effects of the manufacturing processes such as form flexing, welding, and warping because of handling, transporting and installation (Lawson et al. 2014). When components have significant and obvious discrepancies from their originally designed geometry, misfit may occur, resulting in schedule delays, disruption, waste and rework. Finally, the unfavorable and negative effects of component discrepancies can lead to 
uncontrollable and huge project cost increases, as well as a loss in client satisfaction and confidence. While the traditional approach for discrepancy management in construction is to "custom-cut and fit at the job site" (Ballast 2007), the approach often taken in offsite construction is to employ trial-and-error strategies using shim plates, cut-off lengths, trimming \& cutting, etc. Trial-and-error strategies can be prone to error, time consuming, and resource intensive and do not appropriately address the risk associated with the rework.

Offsite construction is similar to the manufacturing industry in many ways, where a high volume of components is produced in an assembly line fashion. In order to control the adverse effects of variability and deviation occurrence in manufacturing, engineers usually use comprehensive tolerance strategies to evaluate critical sources of variability occurrence (Henzold 2006; Hong and Chang 2002). Offsite construction has remained relatively unattended towards or need for systematic tolerancing practices comparing to manufacturing (Johnsson and Meiling 2009; Milberg et al. 2002). Although quality control in offsite construction can be performed more efficiently than in stick-built construction (Isaac et al. 2016), it is often performed using the same traditional approach, which include checklists, material inspections reports and other tools for ensuring that minimum materials and workmanship are met (Thorpe and Sumner 2004). Although systematic tolerancing can be used for controlling the negative impacts of geometric deviation in offsite construction, this approach is not time effective, and requires an extensive prior knowledge into tolerancing principles for proper and effective implementation. Since offsite construction includes the production of numerous exchangeable components, there is a unique opportunity for controlling the negative effects of geometric deviation by planning for an optimal arrangement of components in an assembly.

On the other hand, 3D imaging in construction has been found as a powerful tool for geometric quality monitoring and discrepancy quantification (Nahangi et al. 2015a; Nahangi et al. 2015b). For measuring the incurred discrepancies, 3D point cloud of the as-built state can be registered and compared with the as-designed state represented by 3D CAD models. While the as-designed state of modular components is integrated with the building information model (BIM), the as-built state can be acquired through the use of 3D imaging systems (e.g. laser scanning or digital photogrammetry). The comparison of as-built and as-designed states is not only useful for tracking the as-built state of interchangeable components but can be used for planning the optimum assembly and sequence of interchangeable components.

This paper aims to solve the challenges related to the geometric variability in offsite construction by developing a framework for the determination of the optimal assembly of interchangeable components in an assembly. Instead of designing strict component tolerances or applying systematic tolerancing principles, this paper presents an algorithm to yield a geometrically resilient design that can accept a range of component variability. This is achieved by developing a component aggregation sequence that minimizes rework and therefore increases productivity. Two frameworks are formed for reducing rework: (1) local rework minimization for ensuring optimal component fit, and (2) global rework minimization for ensuring compliance to assembly geometric constraints. The proposed algorithm is demonstrated using a case study. The case study is to plan for serial/parallel assembly of a modular steel bridge. The results of the proposed algorithm show that by planning an optimal arrangement of interchangeable components in a major assembly, rework and waste can be reduced (ideally minimized), while improving productivity and decreasing material handling time. Although the scope of this paper is confined to the aggregation of modular components in an offsite manufacturing facility, it can be applied for optimum planning optimizing of interchangeable modules. 


\section{BACKGROUND}

This section discusses the related research areas to the scope and objective of this paper. Specifically, it is directed toward identifying how sources of variability for the manufacture and aggregation of components in offsite construction assemblies cause rework, unless geometric variability is properly managed. In addition, this section presents an overview of the existing approaches for geometric variability control and optimal aggregation planning in both manufacturing and (offsite) construction.

\section{Types of aggregation in offsite construction}

The process of aggregating components in construction can be defined by two stages: (1) mating, which is to bring components into a rough alignment with each other, and then (2) joining, which is to fix or fasten components together. This is similar to the type of aggregation that occurs for industrial assemblies (Nof et al. 2012).

Two major assembly types in offsite construction are: (1) serial/parallel assemblies, and (2) volumetric assemblies. Serial/parallel assembly originates from robotics and kinematics theory and relates to the aggregation of a single kinematics branch (serial system) or a network of kinematics branches (parallel system), well discussed by (Nahangi et al. 2015a). Serial/parallel assemblies do not make a usable volumetric space. Therefore, they are common in structural or industrial applications (i.e., steel frame structures or pipe spool assemblies) (Feng et al. 2015)jol. Volumetric aggregation originates from manufacturing tolerance theory where aggregation is defined in terms of the interaction of a series of planes and surfaces rather than branches. Ultimately, offsite construction approaches can be described in terms of one distinct type of aggregation (Table 1).

Table 1: Aggregation types within offsite construction, adapted from (Gibb and Isack 2003).

\begin{tabular}{ccc}
\hline Offsite Construction Categories & Sub-categories and Examples & $\begin{array}{c}\text { Aggregation } \\
\text { Type }\end{array}$ \\
\hline \multirow{2}{*}{ Prefabrication } & Components & $\begin{array}{c}\text { Serial-parallel } \\
\text { Volumetric }\end{array}$ \\
\cline { 2 - 3 } & Sub-assemblies & $\begin{array}{c}\text { Serial-parallel } \\
\text { Volumetric }\end{array}$ \\
\hline \multirow{2}{*}{ Preassembly } & Non-volumetric & Serial-parallel \\
\cline { 2 - 3 } & Volumetric & Volumetric \\
\hline Modularization & Single story & Volumetric \\
\cline { 2 - 3 } & Multi-story & Volumetric \\
\hline
\end{tabular}

\section{Impact of geometric variability for assembly aggregation in construction}

In manufacturing, tolerance strategies are commonly used to ensure that components can be properly aggregated into an assembly and form a major assembly. The traditional tolerance strategy lies with past experience, intuition, or by trying to anticipate potential sources of variability and process capabilities (Drake 1999). As such, knowledge about variability sources is required to design effective tolerance and efficient decisions. Sources of variability can be expressed in distinct categories: materials, machines, methods of manufacturing, manpower, measurement, and environment.

Although, high precision manufacturing and aggregation processes have been widely used, some degree of geometric variability in components is inevitable. Even tolerances, which are used in the 
design of offsite components, can accumulate, further causing more profound geometric variability problem. If geometric variability is not managed and handled appropriately, components cannot be assembled, requiring rework to fit the components into correct alignment. Such rework related to geometric variability in offsite construction includes expensive and time consuming field modifications, schedule delays, design compromises, and even functional or serviceability related building failures (Ballast 2007).

\section{Control of geometric variability}

In order to control the impacts of geometric deviations of assemblies in manufacturing, three different approaches can be taken: (1) design for manufacture (DfM), (2) design for assembly (DfA) and (3) design for manufacture and assembly (DfMA). In DfM, components are designed to be manufactured cost-effectively, with less focus on the cost-related aspect of aggregation and assembly. In order to control geometric variability using DfM, it must be performed during the assembly process using techniques such as selective assembly. In DfA, components are designed to be assembled cost-effectively, with less focus on the cost-related aspect of manufacturing. In order to control geometric variability in DfA, components must be extensively toleranced, usually at the expense of expensive and time consuming manufacturing techniques. DfMA is a trade-off approach for minimizing overall production costs by considering the relationship between DfM and DfA (Kamrani and Sa'ed 2002). DfMA is flexible regarding where and how geometric variability is controlled. Rather than placing strict controls on manufacturing or aggregation processes, DfMA can be used to target critical aspects of geometric variability. As such, this design approach is commonly preferred over DfM or DfA for its flexibility and ability to optimize overall production costs. Therefore, this paper presents an automated approach for determining an optimal assembly plan by addressing geometric variability in interchangeable components.

\section{Knowledge gap and research contribution}

This paper presents a framework for optimum assembly planning of interchangeable components in modular construction assemblies. Addressing the challenges related to the minimization of rework in offsite facilities and on construction sites is the ultimate goal of this work. When interchangeable modular segments are being installed and erected on construction sites, there are multiple ways to assemble the components. Furthermore, due to inevitable component geometric variability, tracking the as-built state and updating the assembly plan becomes even more challenging. Finding an assembly plan with minimum geometric deviation from the as-designed state is the key to minimizing rework related to geometric variability. 3D imaging is used to acquire the as-built status of construction assemblies. In this way, the geometric deviations are systematically controlled and therefore the rework associated with such deviations are minimized.

\section{METHODOLOGY}

For automated and optimum assembly planning, 3D imaging is employed as a tool for the representation of the as-built state. In this paper, laser scanning is used for data acquisition in the form of point clouds which are then imported as an input to the processing framework for calculating the optimum assembly plan. As seen in Figure 1, the proposed framework has three primary steps: (1) analyzing modular segments locally, (2) matching the segments globally, and (3) optimizing the assembly plan by minimizing the resulting geometric deviations. By minimizing geometric deviations, aggregation and erection costs are saved and schedule delays are minimized. Each step is discussed extensively in the following sections. 


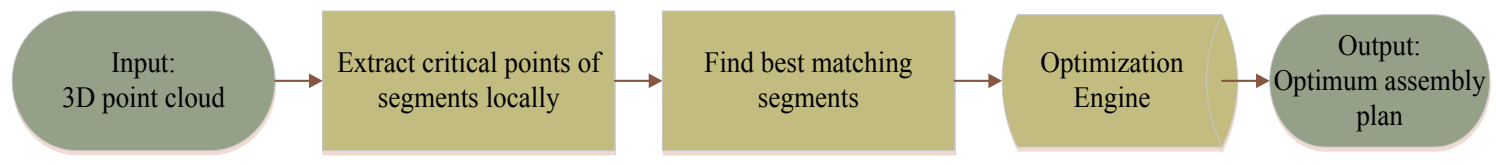

Figure 1: Framework for optimum assembly planning

\section{Identification of critical interface points in local coordinate system}

The global control points are first initialized from the design model. Then, critical interface points are extracted manually from the as-built state represented by an automatically acquired point clouds. An auxiliary software for point cloud manipulation is used for extracting the critical points from 3D point clouds. The coordinates of critical interface points in the local coordinate system in which they were scanned are extracted and stored in an array for further manipulation and required calculations.

\section{Calculation of transformations for matching segments}

Once tie-in and control points for each segment are identified, the required transformation from local to global coordinate system must be calculated. A similar approach suggested by (Kim et al. 2013; Nahangi and Haas 2014) is used here for calculating this transformation. This transformation from local to global coordinate system is denoted by $\left[{ }_{l}^{G} T\right]$, as shown in Figure 2. $\left[{ }_{l}^{G} T\right]$ is then applied as follows:

$$
\{P\}_{G}:=\left[{ }_{l}^{G} T\right]\left\{P_{i}\right\}_{l}
$$

In which, $\left\{P_{i}\right\}_{l}$ is the point set that represents the tie-in points in the local coordinate system, and $\{P\}_{G}$ is the point set in the global coordinate system that matches $\left\{P_{i}\right\}_{l}$.

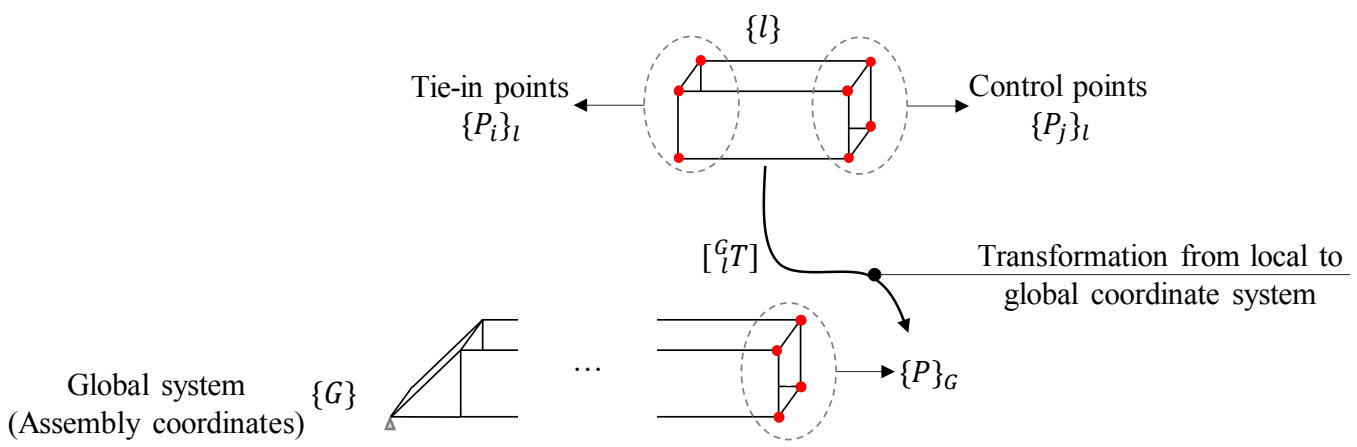

Figure 2: Parameters for transformation of modular components from local to global coordinate system.

As a homogeneous transformation, $\left[{ }_{l}^{G} T\right]$ consists of a rotational $(\vec{R})$ and a translational $(\vec{T})$ part. For calculating the rotation and translation matrix Principal Component Analysis (PCA) is used. PCA matches the points by aligning the principal axes of the point sets. For the PCA alignment, a four-step algorithm should be applied on the datasets:

1- Calculate covariance of the critical point sets in the local and global coordinate systems,

2- Compose singular value decomposition (SVD) of the covariance matrices,

3- Calculate the rotation matrix from the SVD,

4- Rotate the local point set and calculate the translation matrix by comparing the centroids. 
The optimization strategy is explained in the following section.

\section{Optimization strategy}

The next step for optimum assembly planning is to find the best sequence for aggregating and erecting modular components. For this purpose, two strategies are proposed: (1) minimizing rework of the final assembly by finding the sequence of components for each slot that minimizes the geometric deviation at the end point of assembly, and (2) avoiding rework that finds the best component for each segment that minimizes the geometric deviation of critical points for each slot. First approach finds the best assembly order which results in a final rework or deviation minimization, while the latter finds the best component for each slot for avoiding rework occurrence proactively. These strategies are briefly illustrated in Figure 3 and extensively described in the following sections.

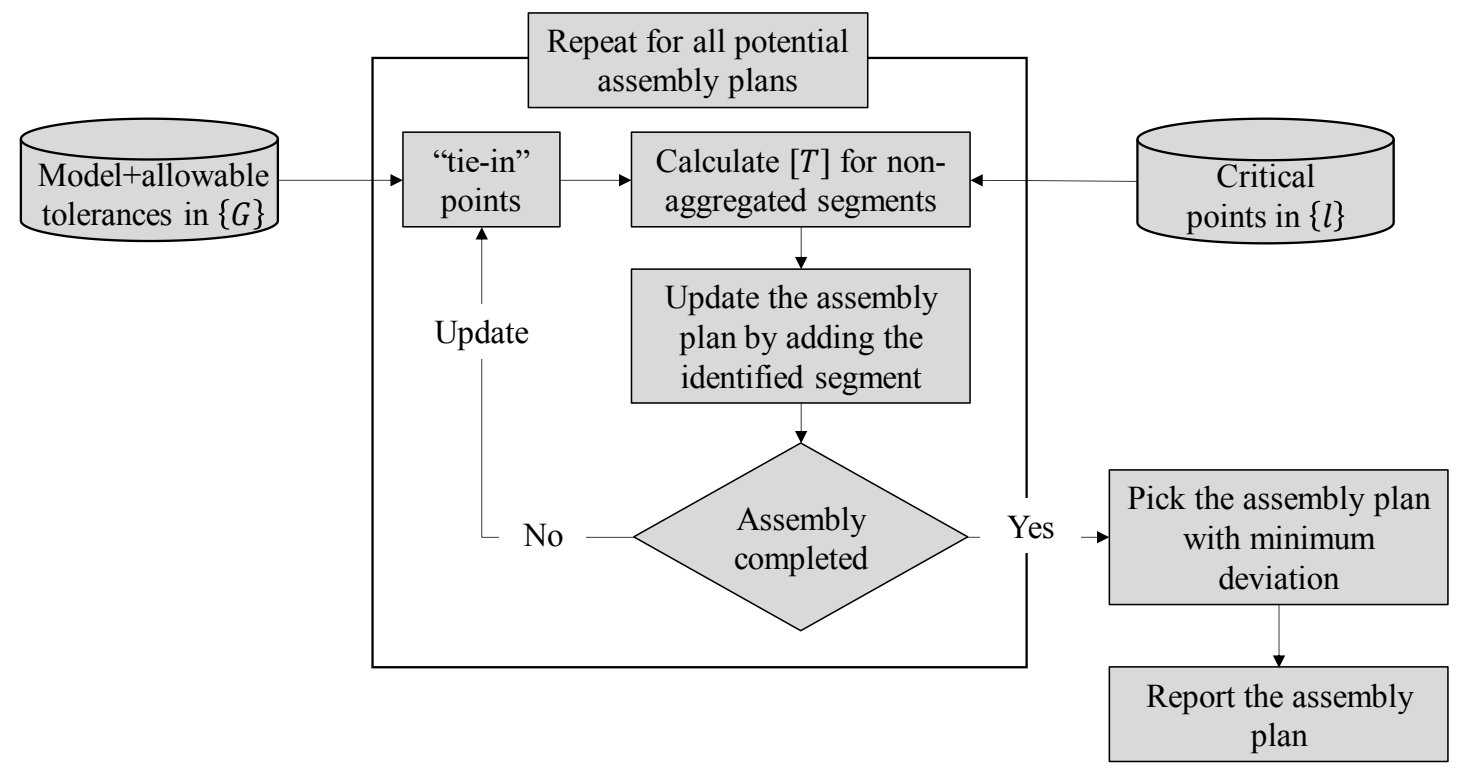

Figure 3: Optimization strategy for assembly planning. This approach finds all potential assembly plans and picks the plan with least resulting deviation.

\section{CASE STUDIES AND RESULTS}

A small scale bridge (illustrated in Figure 4) was used to validate the optimization framework for optimum assembly planning. The bridge was designed in three types of modules (nine modules in total) that are bolted together into a parallel system. The bridge contains six Type 1 modules (Figure 4-b) and one Type 2 (Figure 4-c). The third module type (legs at the ends) was not considered in this case study for simplifying the optimization problem.

The bridge was aggregated into Type 1 and Type 2 modules. Although the six Type 1 modules should be theoretically interchangeable, fabrication process capabilities introduced geometric variability, impacting the degree of interchangeability. As a result, depending on the specific module assembly plan, gaps can be introduced between interfaces, causing the bridge to have different overall lengths. 


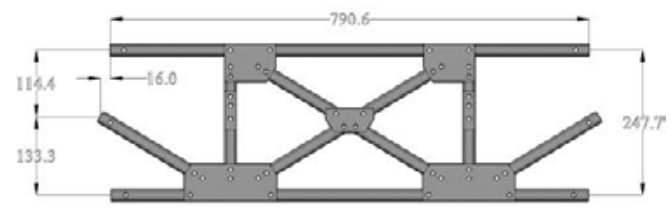

(c) Module type 2

(a)

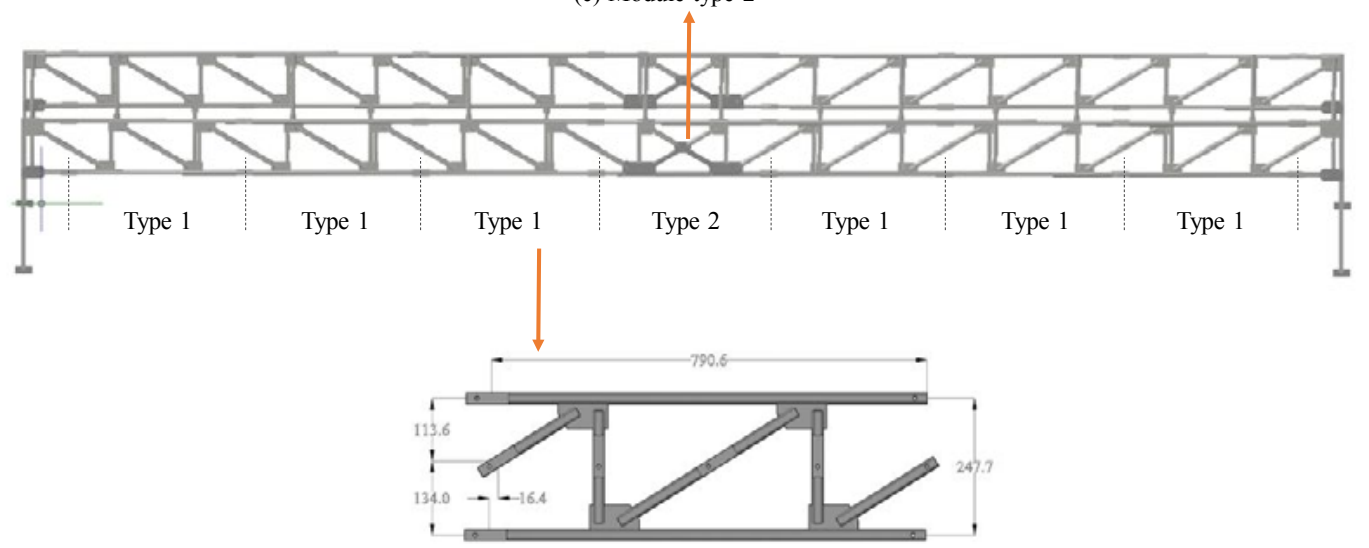

(b) Module type 1

Figure 4: Case Study. (a) 3D model the assembled bridge, geometric dimensions of modules Type 1 (b) and Type 2 (c). Dimensions are in $\mathrm{mm}$.

To collect accurate as-built data, a laser scanner (Faro Edge Arm). The laser scanner used in this case study can scan an object with an accuracy between $0.024 \mathrm{~mm}$ to $0.064 \mathrm{~mm}$ depending on the given working length. In addition, a laser line probe with an accuracy of $0.025 \mathrm{~mm}$ was utilized to create point clouds in a timelier manner than probing individual points. It should be noted that for the laser scanner employed in this case study, two approaches can be taken for acquiring critical interface points:

1- laser scanning each module and manually extracting critical points, or

2- probing the critical interface points directly using the laser line probe. For the purpose of executing the proposed algorithms, probing critical points is efficient, however laser scanning offers a much richer set of data, which can be used for further applications.

Once the critical points are extracted, the implemented optimization approaches applied on the modular bridge components. Both approaches are applicable on the modular bridge as the geometric deviation at each slot or the final segment may be critical. Results are summarized in Table 2 .

Table 2: Optimum assembly planning

\begin{tabular}{|l|l|l|}
\hline Assembly plan & Deviation (mm) & $\begin{array}{l}\text { Processing } \\
\text { time (sec) }\end{array}$ \\
\hline$A P:\{6,2,1,4,7,5,3\}$ & 13.15 & 0.51 \\
\hline
\end{tabular}

\section{CONCLUSIONS}

The key findings of this research are summarized as follows:

- Developing an optimal assembly plan for interchangeable components in cases where geometric deviations create problems for component assembly. 
- Minimizing the overall assembly geometric variation and overall rework by optimally matching critical interface coordinates of components in assemblies.

- Quantifying of expected geometric deviations for local component assembly or the overall assembly discrepancy which can be used for making critical geometric decisions.

Future work includes the automation of the manually performed steps in this framework. For example, automatic extraction of critical points by combining them with the building information models can be an extension to this research.

\section{REFERENCES}

Ballast, D. K. (2007). Handbook of Construction Tolerances, John Wiley \& Sons.

Drake, P. J. (1999). Dimensioning and Tolerancing Handbook, McGraw-Hill New York.

Feng, C., Xiao, Y., Willette, A., McGee, W., Kamat, V. R. (2015). "Vision Guided Autonomous Robotic Assembly and as-Built Scanning on Unstructured Construction Sites." Autom. Constr., 59, 128-138.

Gibb, A., and Isack, F. (2003). "Re-Engineering through Pre-Assembly: Client Expectations and Drivers." Build. Res. Inf., 31(2), 146-160.

Henzold, G. (2006). Geometrical Dimensioning and Tolerancing for Design, Manufacturing and Inspection: A Handbook for Geometrical Product Specification using ISO and ASME Standards, Butterworth-Heinemann.

Hong, Y., and Chang, T. (2002). "A Comprehensive Review of Tolerancing Research." Int J Prod Res, 40(11), 2425-2459.

Isaac, S., Bock, T., Stoliar, Y. (2016). "A Methodology for the Optimal Modularization of Building Design." Autom. Constr.

Johnsson, H., and Meiling, J. H. (2009). "Defects in Offsite Construction: Timber Module Prefabrication." Constr. Manage. Econ., 27(7), 667-681.

Jolliffe, I. (2002). Principal Component Analysis, Wiley Online Library.

Kamrani, A. K., and Sa'ed, M. S. (2002). Product Design for Modularity, Springer Science \& Business Media.

Kim, C., Son, H., Kim, C. (2013). "Fully Automated Registration of 3D Data to a 3D CAD Model for Project Progress Monitoring." Autom. Constr., 35, 587-594.

Lawson, M., Ogden, R., Goodier, C. (2014). Design in Modular Construction, CRC Press.

Milberg, C. (2007). "Tolerance considerations in work structuring." Proc., Proceedings IGLC, 233-243.

Milberg, C., Tommelein, I. D., Alves, T. (2002). "Improving design fitness through tolerance analysis and tolerance allocation." Proc., 3rd International Conference on Concurrent Engineering in Construction, University of California, Berkeley.

Nahangi, M., Czerniawski, T., Haas, C. T., Walbridge, S., West, J. (2015a). "Parallel Systems and Structural Frames Realignment Planning and Actuation Strategy." J. Comput. Civ. Eng.

Nahangi, M., Yeung, J., Haas, C. T., Walbridge, S., West, J. (2015b). "Automated Assembly Discrepancy Feedback using 3D Imaging and Forward Kinematics." Automation in Construction, 56, 36-46.

Nahangi, M., and Haas, C. T. (2014). "Automated 3D Compliance Checking in Pipe Spool Fabrication." Advanced Engineering Informatics, 28(4), 360-369.

Nof, S. Y., Wilhelm, W., Warnecke, H. (2012). Industrial Assembly, Springer Science \& Business Media.

Thorpe, B., and Sumner, P. (2004). Quality Management in Construction, Gower Publishing. 